\title{
Robot Acceptance at Work: A Multilevel Analysis Based on 27 EU Countries
}

\author{
Tuuli Turja $^{1}$ (D) Atte Oksanen ${ }^{1}$ (D)
}

Accepted: 25 January 2019 / Published online: 7 February 2019

(c) The Author(s) 2019

\begin{abstract}
Robots are increasingly being used to assist with various tasks ranging from industrial manufacturing to welfare services. This study analysed how robot acceptance at work (RAW) varies between individual and national attributes in EU 27. Eurobarometer surveys collected in $2012(n=26,751)$ and $2014(n=27,801)$ were used as data. Background factors also included country-specific data drawn from the World Bank DataBank. The study is guided by the technology acceptance model and change readiness perspective explaining robot acceptance in terms of individual and cultural attributes. Multilevel studies analysing cultural differences in technological change are exceptionally rare. The multilevel analysis of RAW performed herein accounted for individual and national factors using fixed and random intercepts in a nested data structure. Individuallevel factors explained RAW better than national-level factors. Particularly, personal experiences with robots at work or elsewhere were associated with higher acceptance. At a national level, the technology orientation of the country explained RAW better than the relative risk of jobs being automated. Despite the countries' differences, personal characteristics and experiences with robots are decisive for RAW. Experiences, however, are better enabled in countries open to innovations. The findings are discussed in terms of possible mechanisms through which the technological orientation and social acceptance of robots may be related.
\end{abstract}

Keywords Change readiness $\cdot$ Europe $\cdot$ ICT exports $\cdot$ Multilevel analysis $\cdot$ Robot acceptance at work $\cdot$ Technology acceptance

\section{Introduction}

For 60 years, industrial robots have gained traction in assembly lines, from precise manufacturing to automotive and food industries [1, p. 1]. Robots that are mechatronic, reprogrammable, and often multidimensional devices are increasingly utilised in various service tasks. In the European Union (EU), Germany stands out as the country with the most industrial robots, although the frequency of service robots is more widespread among European countries [2]. The most common forms of service robots are found in the fields of agriculture, military, medical care, and logistics [3, 4]. However, no robust statistics are available for the number

Tuuli Turja

tuuli.turja@tuni.fi

Atte Oksanen

atte.oksanen@tuni.fi

1 Faculty of Social Sciences, Tampere University, Kalevantie 5, 33014 Tampere, Finland of service robots used in different domains of work. One exception is the milking robot, which is the most common type of service-work robot [4]. The leading EU countries in milking-robot use are Finland, Sweden, and Germany [5].

A basic principle holds that automation offers its best productivity gains when robots replace human work in high-wage countries [6]. For some tasks, human labour is replaced by automation, but technology is also seen as creating a new kind of work, especially in low-end and high-end skills [7-10]. The skill-biased technical change hypothesis considers that automation will lead to higher demands for high-skilled jobs [7-9]. Indeed, studies show that automation risk primarily concerns the low-skill and low-wage range of work. In the service sector, this type of work includes, for example, telemarketing [11]. Nevertheless, organisations may be able to fully capitalise on emerging new technology only if staff members are motivated and accepting of this change. Social acceptance is essential, especially given that social robots will be working alongside people in the same space [12]. 
Robot acceptance at work (RAW) is one of the thresholds of current technological advancements. The U.S. National Robotics Initiative [13] and Japan's Robot Revolution Initiative [14] promote robot use in daily life, as well as higher acceptance of both industrial and service robots. The EU has also taken initiative and funded SPARC, the largest research and innovation programme in civilian robotics in the world [15]. However, do these policies manifest themselves in terms of robotisation readiness in the population?

With no studies exploring national variation in robot acceptance, cross-national comparative studies analysing emerging technological change are currently needed. Due to rapid robotisation in the service sectors, this kind of research evidence is important in understanding the phenomenon in different countries and could provide further necessary information for policymakers, employers, and organisations. Contributing to geographical and cultural discussions of technology, this is the first multilevel study of robot acceptance across the EU population.

The technology acceptance model (TAM) and literature on the readiness for change provide a foundation for explaining RAW with individual experiences and social norms. The robot assistance in question does not segregate industrial and service robots. However, this study is particularly relevant in a time when robots are being increasingly implemented in services. Working with and for people, robots must be accepted by the staff before they can truly be considered as a part of daily routines [16].

\section{Background}

The TAM seeks to understand how users both accept and start to use technology [17]. It is grounded in the theory of reasoned action (TRA), set out in Fishbein and Ajzen [18], and its extension, the theory of planned behaviour (TPB), found in Ajzen [19]. The TAM simulates the way people behave based on their attitudes and intentions. The first version of the TAM neglects the components of social influence included in the TRA and TPB [17]. Subsequent modifications to the TAM have since returned to the original TRA and TPB by explaining perceived usefulness-not only with individual but also social factors [20, 21]. The TAM and its modifications have been widely used to predict the perceived usefulness and usage intentions of technology by individual factors, such as previous experiences and social factors, including subjective norms [22].

Although the TAM focusses on technology itself, we need to expand our analysis to change readiness given that robots are only just arriving to service fields of work. Readiness for organisational change is defined as a mindset in which the change itself and the new task demands are perceived as agreeable [23]. On the verge of-for example, robotisation-people already have an idea of the benefits and risks regarding the change [24].

The readiness for change is dependent on individual and organisational factors. In addition, employees' attitudes are sometimes culturally dependent, as concluded by Ronen and Shenkar [25], who clustered countries by attitudinal dimensions, such as technological development. Ronen and Shenkar [25] divided European countries into five clusters: Latin, Germanic, Anglo, Near Eastern, and Nordic. In a wider scope of value-based differences, Inglehart and Welzel [26] divided Europe into three clusters: traditionally Catholic, traditionally Protestant, and English-speaking countries. Compared to the secular-rational EU countries, traditionally Catholic Mediterranean countries associate more with conservative values [26] and even tend to value religion over science [27, p. 32].

\subsection{Individual Factors Related to RAW}

In prior studies, younger generations, males, and people with higher education have been shown to accept robots more readily than others [28]. Parallel to those of higher education, higher-level employees are typically more open to technological changes in organisations [29].

User experience is also associated with robot acceptance. Experimental studies have demonstrated that attitudes are more positive after, relative to before, introducing a robot to test subjects [30-32]. More broadly, people who have a high competence in ICT (information and communication technology) are also more likely to accept robot assistance compared to those who are less competent in this area [33]. Again, the findings in technology acceptance are consistent with the literature in change management. As Schyns summarises, early participation leads to higher readiness for change [34].

There are a few special characteristics regarding robot acceptance in a work context. Robots are viewed as more acceptable in manufacturing than, say, healthcare work or education [35]. However, regardless of the field of work, implementing new technology demands organisational changes and change management [36]. Using technologies at work is typically mandatory, rather than voluntary, as it is in a consumer context. As an example of this particularity of the work context, cultural norms are hypothesised as 
having a smaller role in work-related robot acceptance than home-related robot acceptance [37].

\subsection{Cultural Factors Related to RAW}

Most technology acceptance and change readiness models stress the importance of social influence. In the TAM, subjective norm refers to what is perceived to be the shared opinion of the social environment. More specifically, shared norms and changes in group dynamics are found to influence technology resistance [38].

Cultural background has been associated with robot likeability, credibility, and trustworthiness $[39,40]$. The perceived attributes of robots even vary between seemingly similar European countries. For example, the Dutch are more prone to anthropomorphism (i.e. humanising lifeless objects) than Germans [28]. Meanwhile, we found no studies that use technological readiness or orientation as a national or other group-level attribute influencing robot acceptance. Studies have primarily focussed on the characteristics that determine acceptance at an individual level.

Given that technology acceptance is socially constructed $[12,20,21]$, it should be explained by the application of individual and cultural predictors. Technological development within a country is one starting point for cross-national comparison; it is indicated by different factors, including the usage of technology and the level of ICT exports. In addition to individual experiences, national-level technological experience (e.g. cellular phone rate) also predicts technology acceptance [41, 42]. The level of ICT exports in particular refers to high-end skill demands in work [11, 42].

The technological development of a country is also considered to predict robot acceptance and provide high-end jobs $[10,21]$. However, we view robot acceptance from the perspective of occupational change and change readiness. The culture that embraces technological changes may also produce fear of employment - especially with low-end skill tasks-because of the risk of job automation [43]. Hofstede's concept of uncertainty avoidance indicates the degree of tolerance for ambiguity and risk [44]. Uncertainty avoidance varies culturally and regulates, for example, the willingness to adopt new technology [45].

Prior cross-cultural studies seem to have concentrated on the differences in individualistic thinking and behaviour. Compared to Western countries, Asian cultures are seen as more collectivistic, where goals and attitudes are shared with some collectivism. Hence, people mostly conform to the norms of society [46]. However, studies on technology acceptance contradict this assumption. In a meta-analysis of the TAM, Schepers and Wetzels [47] compared Western and non-Western studies, finding that subjective norms have a larger impact on behavioural intention in individualistic Western countries.

\subsection{Hypotheses}

In testing Brown and Venkatesh's [37] assumption, we hypothesised that individual factors play a more significant role in RAW than national factors do.

H1 Age, gender, occupation, employment, Internet use, and robot experience together predict RAW more significantly than national technology orientation.

Next, we concentrated on national factors of technological orientation associated with RAW. Hypothesis 2 and 3 can be perceived as being competitive with each other: Does the population-level attitude towards robots depend more on the country's technological developmental history [10, 21, 25] or predictions of work automation in the future $[7-10,43]$ ?

H2 A higher level of technological development (ICT export rate and cellular phone ratio) correlates positively with RAW.

H3 Job automation risk rate correlates negatively with RAW.

\section{Methods}

This study is based on the Special Eurobarometer "Public attitudes towards robots" data, which were collected in two separate years. Eurobarometers are polls administered by the European Commission since 1974 and are funded by the EU. The polls monitor EU citizens' social and political views and account for phenomena such as robotisation. Attitudes towards robots have been found to be relevant in a time where robots are gradually developed for social, not only industrial, environments and working communities. Representative samples of populations aged 15 or over (ca. $1000 /$ country) are collected, along with face-to-face interviews conducted at the participants' homes in their mother tongues. The sampling is based on a random selection of 
sampling points after stratification by the distribution of the national and regional resident population [48]. Eurobarometer data sets are publicly available via different European data archives.

To analyse the temporal variance, we combined data from the $2012(n=26,751)$ and $2014(n=27,801)$ Eurobarometer waves. Both surveys were structured as face-to-face interviews in EU countries. One country (Croatia) was excluded from the analysis because it only had one measurement point. The aggregate data $(N=53,543)$ of repeated crosssectional measures encompassed 27 countries.

\subsection{Dependent Variable}

RAW was measured by a question on how respondents felt about "having a robot assist [them] at work (e.g. in manufacturing)". The scale for the question ranged from 1 (totally uncomfortable) to 10 (totally comfortable) $(M=6.24$; $S D=3.11$ ). This was one of many questions concerning robots in the 2012 and 2014 Eurobarometer surveys [49], yet it was the only one concerning robots assisting the respondent in his or her own work. The intention to use technology was previously measured with a single item [e.g. 50].

Pictures were shown to respondents: first, a food-processing industrial robot gripper and, second, a drink-serving Care-o-Bot 3 robot (see "Appendix A"). The order of the pictures was not randomised because they were both presented prior to the questions concerning robot assistance. The definition of robots was also used to prime the questions after the example pictures:

A robot is defined as a machine which can assist humans in everyday tasks without constant guidance or instruction, e.g. as a kind of co-worker helping on the factory floor or as a robot cleaner, or in activities which may be dangerous for humans, like search and rescue in disasters. Robots can come in many shapes or sizes and some may be of human appearance. Traditional kitchen appliances, such as a blender or a coffee maker, are not considered as robots.

\subsection{Independent Variables}

Individual-level variables included gender (54.4\% female), age $(M=49.59 ; S D=18.15)$, full-time education completion age $(M o=18)$, dichotomous employment status $(50.9 \%$ not working at the time of the study), dichotomous frequent Internet use at work ( $24.4 \%$ used daily), dichotomous experience with robots at work (5.6\% used a robot), and experience with robots elsewhere (sum variable $0-2$ where $0=$ no experience, $1=$ used a robot in one other context, $2=$ used a robot in two other contexts; $M o=0 ; M=0.07$; $S D=0.27)$. The dichotomisation of the employment status was rationalised for the topic of the study: $30.8 \%$ of the respondents were retired (6.3\% before age 65$), 8.4 \%$ were unemployed, $7.1 \%$ were students, and $6.8 \%$ were homemakers. Internet use at work and experiences with robots were dichotomised because of the unequal distribution of the responses.

National-level variables drawn from the World Development Indicators database [51] included cellular phone ratio, referring to subscriptions per 100 people (range 96.3-160.7\%), and ICT exports, referring to the percentage of exported ICT goods relative to total exported goods (range 1.1-12.7\%). ICT exports consisted of computers and peripheral equipment, communication equipment, consumer electronic equipment, electronic components, and miscellaneous information and technology goods. National-level variables also included job-automation risk rate [11], referring to the amount of jobs at risk of being automated in the country (range 46.7-61.9\%). Some national-level variables of interest were excluded from the final model because of their lack of explanatory power. Regarding technological orientation, these included ICT import rate and the intensity rate of research and development. Regarding general indicators, the excluded variables were unemployment rate and gross domestic product.

\subsection{Statistical Analyses}

To analyse the variance components in acceptance of RAW, we applied a multilevel model to the data (Stata 12.1) and examined explanatory variables at a fixed individual and fixed national level. Random effects control for country and time, which are also seen as possible explanatory variables but are random in the sense that they have several more possible levels than what is sampled in the random collection at hand.

Multilevel modelling is an extension of the standard multiple regression analysis. Here, it accounts for correlated responses at levels where dependencies of observations (individual level) or clustering effects (national level) occur. The rationale for using multilevel modelling is the assumption that data nested within a group tend to be more alike than data from individuals selected at random. Eurobarometer statistics for robot acceptance have not been 
combined with national statistics before this study. In this multilevel design, individual-level phenomena are nested within countries. The analysis progresses in three linear regression models.

The preliminary results are reported as percentages, ranges, modes $(M o)$, means $(M)$, standard deviations $(S D)$, and coefficients of variance between groups $(F)$. Before conducting the multilevel analysis, the data were weighted with the coefficient "W22 Weight EU-27" to adjust each national sample in proportion to its share of the total population. The multilevel analysis is reported as unstandardised regression coefficients $(b)$ and their standard errors $(S E)$. The model fit is reported as the log likelihood and Wald $\mathrm{Chi}^{2}$ test statistics. Also, the coefficients of determination $\left(R^{2}\right)$ and Cohen's $d$ effect sizes for linear models $(d)$ were calculated as post hoc power analyses to the final model. Effect sizes are used in addition to $p$ values to calculate a proportion of variability explained, independent of sample size. Also, to indicate the within-group correlation in the nested data, we report the intraclass correlation (ICC).

\section{Results}

More than $86 \%$ of the respondents reported that they had not used a robot in any context, and $12.5 \%$ had used a robot in some context and $0.9 \%$ in more than one context. About $6 \%$ of the respondents had used a robot at work. This proportion has remained roughly the same between the years 2012 and 2014.

Evaluations of RAW were slightly positive in both of the measurement points (2012: $M=6.30 ; M o=10 ; S D=3.07$ and 2014: $M=6.19 ; M o=10 ; S D=3.16)$. Although the average level of RAW was somewhat decreased over time, the difference was not significant. Means by country and year are in "Appendix B". In 2014, the most positive views were reported in Sweden, Estonia, Denmark, and Poland, with the most negative views in Romania, Cyprus, Portugal, and Greece.

The variance analysis indicated the average RAW scores differing between countries $(F(20)=166.58 ; p<.001)$. Less variance was found for the two time points $(F(1)=8.03$; $p<.005)$, but an interaction between country and time was highly significant $(F(20)=3.76 ; p<.001)$. However, a

Table 1 Multilevel analysis of RAW, linear regression

\begin{tabular}{|c|c|c|c|c|c|c|c|c|}
\hline & \multirow{2}{*}{\multicolumn{2}{|c|}{$\begin{array}{l}\text { Model } 1 \\
\text { National level } \\
(n=49,900)\end{array}$}} & \multirow{2}{*}{\multicolumn{2}{|c|}{$\begin{array}{l}\text { Model } 2 \\
\text { Individual level } \\
(n=50,143)\end{array}$}} & \multirow{2}{*}{\multicolumn{4}{|c|}{$\begin{array}{l}\text { Model } 3 \\
\text { Individual level \& nationallevel } \\
(n=49,204)\end{array}$}} \\
\hline & & & & & & & & \\
\hline & $b$ & $S E b$ & $b$ & $S E b$ & $b$ & $S E b$ & $R^{2}$ & $d$ \\
\hline \multicolumn{9}{|l|}{ Robot acceptance at work (RAW) } \\
\hline \multicolumn{9}{|l|}{ Fixed effects ${ }^{\mathrm{a}}$} \\
\hline \multicolumn{9}{|l|}{ National-level effects } \\
\hline ICT exports & $.108 * * *$ & .022 & & & $.104 * * *$ & .019 & .027 & \\
\hline Cellular-phone ratio & $.020 * *$ & .007 & & & $.017 * *$ & .007 & .013 & \\
\hline Job-automatisation risk & $-.113 * *$ & .038 & & & $-.088^{*}$ & .037 & .021 & \\
\hline \multicolumn{9}{|l|}{ Individual-level effects } \\
\hline Gender (female) & & & $-.617 * * *$ & .088 & $-.617 * * *$ & .088 & & .220 \\
\hline Age & & & -.004 & .003 & -.004 & .003 & .008 & \\
\hline Years of education & & & $.177 * * *$ & .015 & $.177 * * *$ & .015 & .059 & \\
\hline Employed & & & $-.244 * * *$ & .067 & $-.244 * * *$ & .067 & & .123 \\
\hline Daily Internet use at work & & & $.396^{* * *}$ & .098 & $.395 * * *$ & .099 & & .316 \\
\hline Robot experience at work & & & $1.195 * * *$ & .125 & $1.194 * * *$ & .125 & & .518 \\
\hline Robot experience elsewhere & & & $.523 * * *$ & .122 & $.523 * * *$ & .123 & .005 & \\
\hline Constant & $8.334 * * *$ & 2.110 & $6.266 * * *$ & .236 & $8.213 * * *$ & 2.059 & & \\
\hline \multicolumn{9}{|l|}{ Random intercept effects } \\
\hline National-level SD & .604 & .080 & .818 & .121 & .521 & .080 & & \\
\hline Time-level SD & .194 & .064 & .209 & .065 & .210 & .065 & & \\
\hline$S D$ (residual) & 2.910 & .041 & 2.807 & .032 & 2.806 & .032 & & \\
\hline Log likelihood & $133,925.41$ & & $123,178.80$ & & 123,056 & & & \\
\hline Wald chi $^{2}$ & $63.19 * * *$ & & $987.48 * * *$ & & $1089.02 * * *$ & & & \\
\hline
\end{tabular}

${ }^{a}$ Data weighted by EU-27, providing adjustments for each national sample in proportion to its share in the total EU population aged 15 and over $* p<.05 ; * * p<.01 ; * * * p<.001$ 
Tukey's test did not produce any robust groups of countries sharing similar variations in RAW.

A multilevel analysis was employed to analyse RAW at the individual and national levels. Due to the satisfactory national-level differences $(\mathrm{ICC}=.08)$ but ambiguous results in grouping and comparing the countries, we employed multilevel models with country and time added as random effects (Table 1). To the first model, we added national-level fixed effects; to the second model, we added individual-level fixed effects. The third model consisted of national- and individual-level effects.

In the third model, the most variance in RAW was captured by individual-level factors (H1). More acceptance was found in males $(b=-.617)$ who had studied longer $(b=.177)$, were not employed $(b=-.244)$, and had personal experience with robots at work $(b=1.194)$ or elsewhere $(b=.523)$. Average RAW scores were significantly different between those who had robot experience at work and those who did not. The $6 \%$ who had used a robot at work had almost 1.2 points more positive assessments of RAW. Effect size $(d=.518)$ indicates the linear difference between the two groups as more than half the standard deviation. The respondents who were employed and used the Internet daily for work also had higher RAW than those who did not use the Internet daily $(b=-.395)$.

At the national level, more acceptance was found in countries with higher levels of ICT exports $(b=.104)$, more cellular phones per capita $(b=.017)(\mathrm{H} 2)$, and lower jobautomation risk $(b=-.088)(\mathrm{H} 3)$.

The individual and national variance components are the differences in averages on the outcomes across countries and time. In addition, the random part of the model showed considerably more variance between the countries than between the 2 years of measured RAW.

\section{Discussion}

In the first multilevel analysis of robot acceptance, we studied the variance of RAW regarding individual and national attributes in the European population. Based on the TAM $[20,21]$, we expected to find differences, first, among individuals and, second, between country-level technological orientation and job-automation risk rate.

Supporting our first hypothesis and Brown and Venkatesh's [37] study, the individual-level differences accounted for more variance than national-level differences. Those who had used a robot at work felt significantly more comfortable with robot assistance at work, which supports the experimental findings that the introduction of robots leads to better robot acceptance [30-32]. Also, male respondents and those with longer education were more prone to accept robots at work, which matches the findings of past research [28]. However, respondents who were not employed at the time of the study reported a higher level of RAW. This is mostly attributable to the more positive attitudes of students and retired people compared to those employed. As a result, age, at least to some extent, did not significantly explain acceptance in the linear models.

The connection between RAW and technological orientation stems from the data due to diverse factors. At the individual level, those who reported using the Internet daily at work had a higher level of RAW, which is somewhat in line with Katz and Halpern's finding that ICT-competent people are more accepting of robots [33]. The finding is supported by the national-level result of higher RAW in countries with a larger cellular phone ratio. This result supports our hypothesis and can be interpreted as technological readiness in the country being linked to more positive views of robots.

Our study contributes to robot acceptance discussions by introducing a model that includes national variation. The best national explanatory variable was the ICT export rate, which indicates technological development and the level of high-end skilled work within a country. In line with our hypothesis, a higher proportion of ICT goods out of the total exports predicted higher RAW. Also in support of the third hypothesis, lower job-automation risk in a country predicted higher RAW. These results imply that acceptance depends not only on perceived opportunities in high-end skilled work but also perceived opportunities in low-end, skilled, easily automated work.

Unexpectedly, the attitude towards robots was higher in countries with both technological advances and at a lower risk for future work automation. Cellular phone frequency and high-technology exports refer to the near history of the countries' technological orientation. On the other hand, the risk of jobs being replaced by automation refers to the future. Although technological orientation associates positively with RAW, views regarding an automated future have an opposite effect on robot acceptance. RAW was lower in countries facing more automation. Nevertheless, the national orientation in manufacturing and using high technology seems to increase RAW more than the risk of jobs being automated reduces it. Technological development enables a generally optimistic attitude towards robotisation, whereas 
a risk for automatised production is actually a risk for some and an opportunity for others.

As Bartneck et al. [39] and Li et al. [40] have shown, there are cultural differences in robot acceptance. We strived to deepen the knowledge of cultural variability by conducting a multilevel analysis. Differences between EU countries in our data can be considered, first, as a result of the dissimilarities in robot frequency or implementation areas. However, the mechanism of experience leading to acceptance is far from straightforward, for the population in some of the most robotised EU countries (i.e. Germany and Finland) do not appear to be the most accepting of robots. Second, cultures vary in terms of how people are prone to experiencing social robots when it comes to enjoyment, anthropomorphism, and perceived behavioural control [28]. This discussion usually touches upon the differences between Western and Asian cultures [40, 47], but even a smaller scale divergence can make a difference [28], as was the case for our European data. Third, we can look at the variation between countries as an outcome of the national media discourse centring on more positive or negative headlines (e.g. addressing robots and work-related changes). Additionally, the emphasis and possible changes in media discourse offer one possible explanation for the temporal changes within countries.

Comparing the 2 years of data, the evaluations of RAW did not change on average, although there were significant "two-year trends" within countries towards both positive and negative attitudes. The highest upward trends were found in Luxembourg and Austria. The highest downward trends were in the Slovak Republic and Finland. In Luxembourg and Austria, the proportion of the highest paid occupations increased between 2011 and 2013, whereas this proportion decreased in Finland [52]. Hence, in the countries where the average attitude changed the most in a positive direction, work opportunities in high-wage jobs increased. This adds to the association of higher RAW in countries with higher ICT exports, indicating more perceived opportunities at high-end skilled work.

The average levels of RAW in the combined data suggest that the acceptance of robots will be slower in Mediterranean countries compared to other EU countries. The most positive views on RAW came mainly from Northern and Eastern European countries, with the most negative coming from Southern Europe. Of these most rejecting countries, Cyprus, Portugal, Malta, Greece, Italy, and Spain are categorised as traditionally Catholic Mediterranean countries [26] that embody a conservative culture with persistent traditions and ideals that are passed on from one generation to the next [11, $32,53,54]$.
This cultural conventionality may manifest as resistance to new technology in work contexts. However, the differences in RAW are not simply explainable by the rate of technology adoption in countries and in workplaces [42]. Likewise, they are not explainable by a country's profiles in individuality, hierarchicality, uncertainty avoidance [44, 55], or even technological similarity [56].

Nevertheless, the division between Mediterranean countries and others, especially Northern and Eastern European countries, is also reflected in ICT export statistics. Estonia, Hungary, the Netherlands, Poland, and Sweden have the highest ICT export rates in the EU. The mixed profiles of the top five ICT exporters include relatively strong innovator countries, namely Estonia, the Netherlands, and Sweden [57], but also Hungary and Poland, where their high ICT export rates mostly consist of subcontracting electrical equipment $[58$, p. 18). Therefore, we suggest that the mixed country profile regarding both higher RAW and higher ICT exports is a combination of high-skill work centred in ICTinnovating countries and low-skill work centred in countries that benefit from cooperation with ICT-innovating countries.

In a theoretical sense, we claim that Europe is roughly divided between conventional and innovative countries. Innovativeness can refer either to active product development or to investments in robotising production lines. Economic globalisation and the free movement of workers inside the EU will undoubtedly mix conventional and innovative cultures together. From the viewpoint of a multinational company choosing destinations for implementing new and robotised operations, they may look to countries that are proven to have high robot acceptance and an innovative stance on the renewal of work. Nevertheless, it is as much about considering the cultural differences inside a country and inside a workplace for the various cultures to work together. In a practical sense, this means, for example, different approaches in orienting new employees from various cultures.

Regardless of the large and representative statistics used, some data shortages still persist. Eurobarometer surveys only provide population-based samples with no possibility for singling out specific occupational groups. Also, due to the limitations of Eurobarometer data, value-based motives behind robot acceptance were approachable only in the national-level discussion.

Our study has a hypothetical bias because a minority of the respondents have personally used a robot. However, the lack of experience does not take away from the aim of measuring readiness for robotisation [24]. Although most respondents had to imagine a robot and a concomitant 
reaction to said robot, the variance of imagination was minimised by the questionnaire's inclusion of a definition and illustrations of robots. Yet, the study designs aimed at maximising the coefficients of determination would need to consider the robots' attributes as well as the people using them [59]. Also, video examples of robots would have been more explanatory than the pictures used.

\section{Conclusions}

We found that accepting robots as assistants at work is mainly associated with individual experiences. However, national-level attributes related to a country's history of manufacturing high technology goods especially contributed to the total variance. The correlation between RAW and national-level factors leads to the conclusion that social norms further regulate robot acceptance. Here, our study offers a novel aspect to the one-level discussions mostly focussed on the individual factors explaining robot acceptance.

The distribution of RAW did not strictly follow prior models of country differences. Instead, robot acceptance seems to follow a novel divide between conventional countries and innovative countries of either their own product development or production robotisation. Our study implies that robotisation brings challenges depending on the technological developments in each country. In a robotising world, work opportunities may actually decrease in cultures less open to the renewal of work. This will only further the already existent divide between the innovative and noninnovative European countries.

Although the explanatory power at the national level was found to be lower than at the individual level, the results support the multilevel examinations of the nested data. Future studies should investigate how RAW varies between different organisations and groups of professionals.

Acknowledgements This research is part of the project Robots and the Future of Welfare Services (2015-2020), which is funded by the Academy of Finland's Strategic Research Council (Grant Number 292980).

\section{Compliance with Ethical Standards}

Conflict of interest The authors declare that they have no conflict of interest.

Open Access This article is distributed under the terms of the Creative Commons Attribution 4.0 International License (http://creativeco mmons.org/licenses/by/4.0/), which permits unrestricted use, distribution, and reproduction in any medium, provided you give appropriate credit to the original author(s) and the source, provide a link to the Creative Commons license, and indicate if changes were made.

\section{Appendix A}

\section{Illustrations from the Eurobarometer questionnaire}
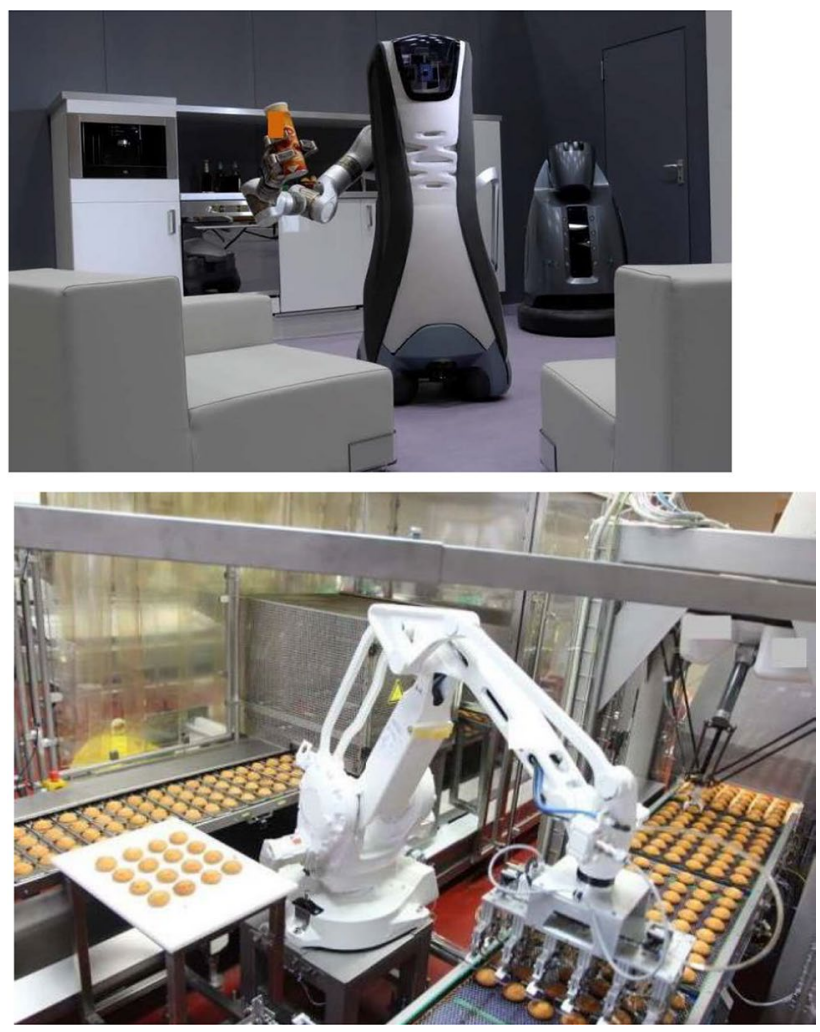


\section{Appendix B}

\section{Robot acceptance at work (RAW) in terms of country and year means}

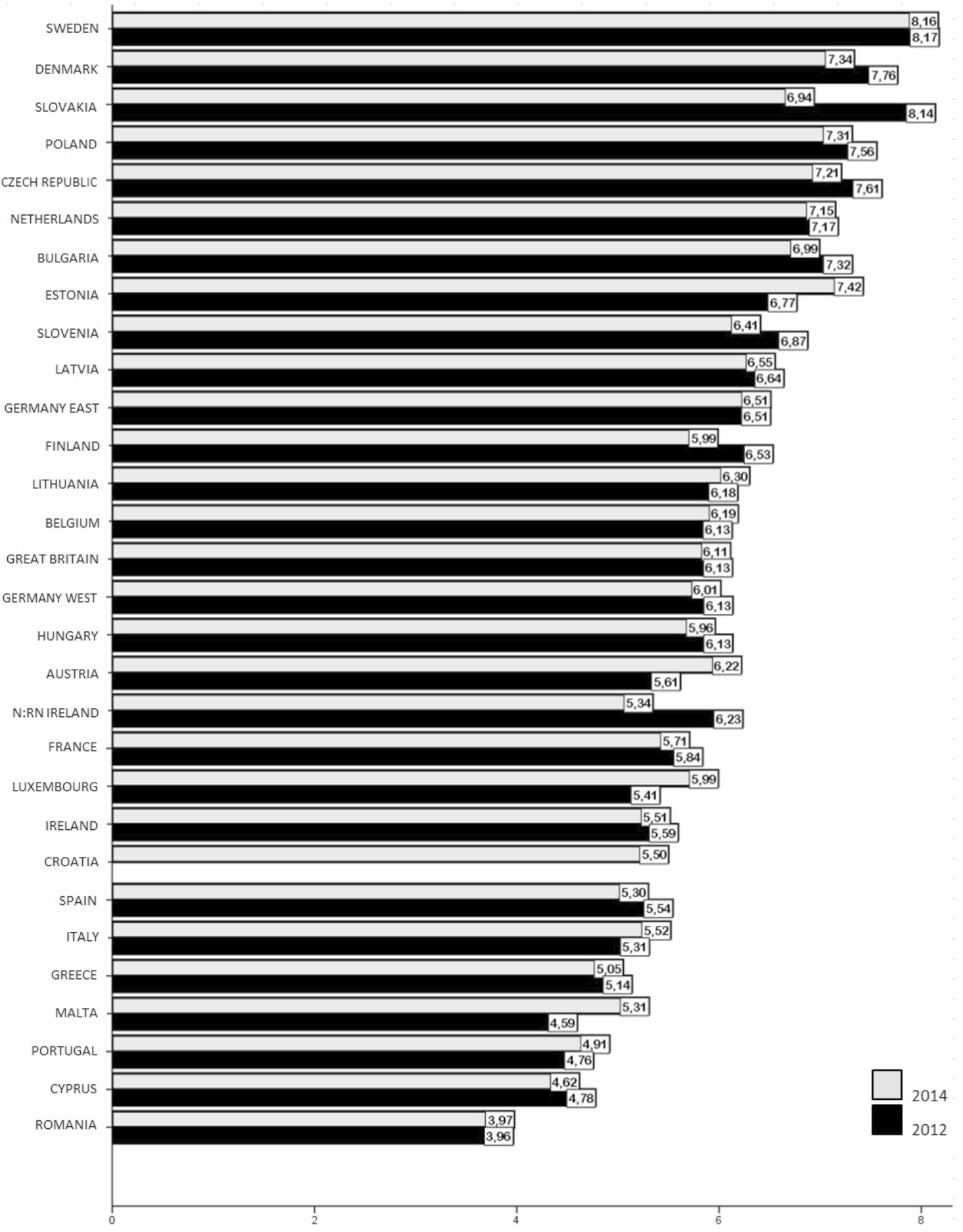




\section{References}

1. Siciliano B, Khatib O (2008) Introduction. In: Siciliano B, Khatib O (eds) Handbook of robotics. Springer, Berlin, pp 1-8

2. European Robotics Association (2015) World robotics. http:// www.eu-nited.net/robotics/news-events/robotics-news/world -robotics-2015.html. Accessed 26 Sept 2017

3. Hagele M (2016) Robots conquer the world [turning point]. Robot Autom Mag 23:118-120

4. IFR (2015) World robotics 2015 service robots. International federation of robotics. http://www.ifr.org/service-robots/statistics . Accessed 27 Sept 2017

5. Rodriguez F (2012) The realities of robotic milking technology today. http://www.progressivedairy.com/topics/management/therealities-of-robotic-milking-technology-today. Accessed $27 \mathrm{Sept}$ 2017

6. Probst LL, Frideres L, Pedersen B, Caputi C (2015) Service innovation for smart industry: human-robot collaboration. European Commission, Luxembourg

7. Acemoglu D (1999) Changes in unemployment and wage inequality: an alternative theory and some evidence. Am Econ Rev 89:1259-1278

8. Acemoglu D (2002) Technical change, inequality, and the labor market. J Econ Lit 40:7-72

9. Autor DH, Levy F, Murnane JR (2003) The skill content of recent technological change: an empirical exploration. Q J Econ 118:1279-1334

10. Fernández-Macías E (2012) Job polarization in Europe? Changes in the employment structure and job quality, 1995-2007. Work Occup 39(2):157-182. https://doi.org/10.1177/073088841142707 8

11. Bowles J (2014) The computerisation of European jobs. http:// bruegel.org/2014/07/the-computerisation-of-european-jobs/. Accessed 11 May 2017

12. Otway HJ, Von Winterfeldt D (1982) Beyond acceptable risk: on the social acceptability of technologies. Policy Sci 14:247-256

13. Huber D, Hebert M (2016) 2015 National robotics initiative PI meeting report. http://nri2015.ri.cmu.edu/wp-content/uploa ds/2016/02/2015-NRI-workshop-report.pdf. Accessed 26 Sept 2017

14. RRI Council (2015) Action plan for FY 2015. https://www.jmfrr i.gr.jp/content/files/20150925_plan_eng.pdf. Accessed 26 Sept 2017

15. Horizon/2020 (2016) Robotics. European commission. http:// ec.europa.eu/programmes/horizon2020/en/h2020-section/robot ics. Accessed 28 Sept 2017

16. Weng Y, Chen C, Sun C (2009) Toward the human-robot co-existence society: on safety intelligence for next generation robots. Int J Soc Robot 1:267-282. https://doi.org/10.1007/s12369-009-0019-1

17. Davis FD, Bagozzi RP, Warshaw PR (1989) User acceptance of computer technology: a comparison of two theoretical models. Manag Sci 35:982-1003. https://doi.org/10.1287/mnsc.35.8.982

18. Fishbein M, Ajzen I (1975) Belief, attitude, intention, and behavior: an introduction to theory and research. Addison-Wesley, Reading

19. Ajzen I (1985) From intentions to actions: a theory of planned behavior. In: Kuhl J, Beckmann J (eds) Action control: from cognition to behavior. Springer, New York, pp 11-39

20. Malhotra Y, Galletta DF (1999) Extending the technology acceptance model to account for social influence: theoretical bases and empirical validation. In: Proceedings of the 32nd Hawaii international conference on system science, pp 1-14. http://doi. org/10.1109/hicss.1999.772658
21. Venkatesh V, Davis FD (2000) A theoretical extension of the technology acceptance model: four longitudinal field studies. Manag Sci 46:186-204. https://doi.org/10.1287/mnsc.46.2.186.11926

22. Marangunić N, Granić A (2015) Technology acceptance model: a literature review from 1986 to 2013. Univers Access Inf 14:81-95

23. Frese M, Zapf D (1994) Action as the core of work psychology: a German approach. In: Triandis HC, Dunette MD, Hough LM (eds) Handbook of industrial and organizational psychology. Consulting Psychologists, Palo Alto, pp 271-346

24. Prochaska JO, Redding CA, Evers K (1997) The transtheoretical model of change. In: Glanz K, Lewis FM, Rimer BK (eds) Health behaviour and health education. Jossey-Bass, San Francisco, pp $60-84$

25. Ronen S, Shenkar O (1985) Clustering countries on attitudinal dimensions: a review and synthesis. Acad Manag Rev 10:435-454

26. Inglehart R, Welzel C (2014) The WVS cultural map of the world. World value surveys. http://www.worldvaluessurvey.org/WVSNe wsShow.jsp?ID=192. Accessed 17 Dec 2017

27. Engelbrekt K, Nygren B (2014) Russia and Europe: building bridges, digging trenches. Routledge, New York

28. de Graaf M, Ben Allouch S (2013) Exploring influencing variables for the acceptance of social robots. Rob Auton Syst 61:1476-1486

29. Waddell D, Sohal AS (1998) Resistance: a constructive tool for change management. Manag Decis 36(8):543-548

30. Heerink M (2011) Exploring the influence of age, gender, education and computer experience on robot acceptance by older adults. In: The sixth ACM/IEEE international conference on humanrobot interaction. Lausanne

31. Louie WYG, McColl D, Nejat G (2014) Acceptance and attitudes toward a human-like socially assistive robot by older adults. Assist Technol 26(3):140-150. https://doi.org/10.1080/10400 435.2013.869703

32. Nomura T, Kanda T, Suzuki T (2006) Experimental investigation into influence of negative attitudes toward robots on human-robot interaction. AI Soc 20(2):138-150. https://doi.org/10.1007/s0014 6-005-0012-7

33. Katz JE, Halpern D (2014) Attitudes towards robots suitability for various jobs as affected robot appearance. Behav Inf Technol 33(9):941-953. https://doi.org/10.1080/0144929X.2013.783115

34. Schyns B (2004) The influence of occupational self-efficacy on the relationship of leadership behavior and preparedness for occupational change. J Career Dev 30:247-261

35. Taipale S, Luca FD, Sarrica M, Fortunati L (2015) Robot shift from industrial production to social reproduction. In: Vincent $\mathrm{J}$, Taipale S, Sapio B, Lugano G, Fortunati L (eds) Social robots from a human perspective. Springer, London, pp 11-24

36. Willcocks L, Lester S (1994) Evaluating the feasibility of information systems investments: recent UK evidence and new approaches. In: Willcocks L (ed) Information management. Springer, New York, pp 49-77

37. Brown SA, Venkatesh V (2005) Model of adoption of technology in households: a baseline model test and extension incorporating household life cycle. MIS Q 29:399-426

38. Chen NH, Huang CT (2016) Domestic technology adoption: comparison of innovation adoption models and moderators. Hum Factors Ergon Manuf 26:177-190

39. Bartneck CT, Suzuki T, Kanda T, Nomura K (2007) The influence of people's culture and prior experiences with Aibo on their attitude towards robots. AI Soc 21:217-230

40. Li D, Rau PLP, Li Y (2010) A cross-cultural study: effects of robot appearance and task. Int J Soc Robot 2:175-186

41. Yoo SJ, David Huang W (2011) Comparison of web 2.0 technology acceptance level based on cultural differences. J Educ Technol Soc 14(4):241-252

42. Berger T, Frey C (2016) Structural transformation in the OECD: digitalisation, deindustrialisation and the future of work. OECD 
Social, Employment and Migration Working Papers. No. 193, OECD Publishing, Paris

43. Saner M, Wallach W (2015) Technology unemployment, AI, and workplace standardization: the convergence argument. J Evol Technol 25(1):74-80

44. Hofstede G (2001) Culture's consequences: comparing values, behaviors, institutions, and organizations across nations. Sage, London

45. Ashraf AN, Thongpapanl N, Auh S (2014) The application of the technology acceptance model under different cultural contexts: the case of online shopping adoption. Int J Mark Stud 22(3):68-93

46. Triandis H (1988) Collectivism v. individualism: a reconceptualisation of a basic concept in cross-cultural social psychology, cross-cultural studies of personality, attitudes and cognition. In: Bagley C, Verma GK (eds) Personality, cognition and values: cross-cultural perspectives of childhood and adolescence. Macmillan, London, pp 60-95

47. Schepers J, Wetzels M (2007) A Meta-analysis of the technology acceptance model: investigating subjective norm and moderation effects. Inf Manag 44:90-103

48. Eurobarometer data service (2017) Sampling and fieldwork. https ://www.gesis.org/eurobarometer-data-service/survey-series/stand ard-special-eb/sampling-and-fieldwork. Accessed 22 Feb 2018

49. Eurobarometer (2016) Public attitudes towards robots. Gesis database. http://dx.doi.org/10.4232/1.12265 Accessed 15 Mar 2016

50. Schnall R, Bakken S (2011) Testing the technology acceptance model: HIV case managers' intention to use a continuity of care record with context-specific links. Inform Health Soc Care 36(3):161-172

51. World Bank DataBank (2016) World development indicators database. http://databank.worldbank.org/data. Accessed 3 May 2016

52. Fernández-Macías E (2015) Job polarisation in Europe: are midskilled jobs disappearing? https://www.socialeurope.eu/2015/07/ job-polarisation-in-europe-are-mid-skilled-jobs-disappearing. Accessed 28 Sept 2017

53. Brey PW, Reijers W, Rangi S, Toljan D, Romare J, Collste G (2015) International differences in ethical standards and in the interpretation of legal frameworks. http://satoriproject.eu/media /D3.2-Int-differences-in-ethical-standards.pdf. Accessed 16 Dec 2017

54. Inglehart R, Baker WE (2000) Modernization, cultural change, and the persistence of traditional values. Am Sociol Rev 65:19-51

55. Hofstede G (2016) Country comparison database. https://geert -hofstede.com/countries.html. Accessed 28 Nov 2017

56. Breschi S, Tarasconi G (2013) The technological profile and specialization pattern of countries. https://ec.europa.eu/research/ innovation-union/pdf/technological-specialization-of-countries. pdf. Accessed 22 Feb 2018

57. Veugelers R (2016) The European Union's growing innovation divide. Bruegel policy contribution (08). http://bruegel.org/wpcontent/uploads/2016/04/pc_2016_08.pdf. Accessed 22 Feb 2018

58. International Trade Statistics (2015) World trade organization. https://www.wto.org/english/res_e/statis_e/its2015_e/its2015_e. pdf. Accessed 20 Dec 2017

59. Hancock PA, Billings DR, Schaefer KE, Chen JYC, de Visser EJ, Parasuraman R (2011) A meta-analysis of factors affecting trust in human-robot interaction. Hum Factors 53(3):517-527

Publisher's Note Springer Nature remains neutral with regard to jurisdictional claims in published maps and institutional affiliations.

Tuuli Turja is a researcher (BA Psych, MSocSci) at the Tampere University. In the project Robots and the Future of Welfare Services she has studied robotization readiness, technology-mediated interaction and motivational technology acceptance particularly in care work.

Atte Oksanen (Dr. Soc. Sci.) is professor of social psychology at the Tampere University. Oksanen's research focuses on emerging technologies and social interaction. He has published in a variety of areas including youth studies, drug and alcohol research and criminology. 